\title{
Corrigendum: Vectorial optical field reconstruction by attosecond spatial interferometry
}

P. Carpeggiani, M. Reduzzi, A. Comby, H. Ahmadi, S. Kühn, F. Calegari, M. Nisoli, F. Frassetto, L. Poletto, D. Hoff, J. Ullrich, C. D. Schröter, R. Moshammer, G. G. Paulus and G. Sansone

Nature Photonics 11, 383-389 (2017); published online 29 May 2017; corrected after print 29 June 2017.

In the version of this Article originally published, the following sentences were missing from the Acknowledgements: "Financial support by the Alexander von Humboldt Foundation (Project Tirinto) and the Italian Ministry of Research (project FIRB no. RBID08CRXK) is gratefully acknowledged. A.C. acknowledges financial support from ENS Paris-Saclay." These have now been added in the online versions of the Article. 